\title{
Generalized Dold sequences on partially-ordered sets*
}

\author{
Grzegorz Graff
}

Faculty of Applied Physics and Mathematics,

Gdańsk University of Technology,

Narutowicza 11/12, 80-233 Gdańsk, Poland

Orcid: 0000-0001-5670-5729

grzegorz.graff@pg .edu.pl

Jacek Gulgowski

The Faculty of Mathematics, Physics and Informatics, University of Gdańsk,

Wita Stwosza 57, 80-952 Gdańsk, Poland

Orcid: 0000-0002-7706-9263

dzak@mat.ug.edu.pl

Małgorzata Lebiedź

The Faculty of Mathematics, Physics and Informatics, University of Gdańsk,

Wita Stwosza 57, 80-952 Gdańsk, Poland

Orcid: 0000-0002-4985-8249

malgosilla@gmail.com

Submitted: Jul 5, 2021; Accepted: Jan 11, 2022; Published: Feb 11, 2022

(C) The authors. Released under the CC BY-ND license (International 4.0).

\begin{abstract}
Dold sequences constitute an important class of integer sequences that play an important role in combinatorics, number theory, topology and dynamical systems. We generalize the notion of Dold sequence for the case of partially ordered sets and describe their properties. In particular we give two alternative descriptions of generalized Dold sequences: by some class of elementary sequences as well as by different generalized arithmetical functions, both defined on a partially ordered set. We also define vector Dold sequences and show their combinatorial interpretation in terms of periodic points.
\end{abstract}

Mathematics Subject Classifications: 11B50, 05A10, 37C25, 55M20

*Research supported by the National Science Centre, Poland, within the grant Sheng 1 UMO2018/30/Q/ST1/00228. 


\section{Introduction}

Dold sequences are an important class of integer sequences satisfying some congruences (Definition 1). They recently have attracted growing attention due to the fact that they constitute a bridge joining various mathematical fields: combinatorics and number theory with topology and dynamical systems.

Let us mention here that there is an ambiguity in terminology, which results from the fact that Dold sequences (we follow the nomenclature from [24]) appear in so many contexts (cf. review article [6]). They are known under different names such as: pre-realizable sequences [32], relatively realizable sequences [28], Gauss sequences [40], generalized Fermat sequences [10] and Fermat sequences [11].

One of the important results, that has a strong impact on periodic point theory, was finding that the sequence of fixed point indices of iterations is a Dold sequence (Dold, [8]). The theorem opened a new field of research in which the influence of the form of indices of iterations on the structure of periodic points and the dynamical behaviour of the map was examined. This brought about a series of new results related to (among other) smooth maps [7, 15, 16], planar homeomorphisms [17, 21, 25], 3-dimensional homeomorphisms [20, 22], simplicial maps [13].

Let us mention that the sequence of Lefschetz numbers of iterations $\left(L\left(f^{n}\right)\right)_{n}$ is an another example of Dold sequence, which results straightforwardly from the fact that the sequence of traces of the powers of an integer-valued matrix is a Dold sequence $[19,40]$.

Another method of creating Dold sequences is given by generating sequence for Newton sequences. The last class of sequences is defined on the basis of Newton identities [26]. Let us remind that the sequence $\left(a_{n}\right)_{n}$ is generated by the generating sequence $\left(c_{n}\right)_{n}$ if:

$$
a_{n}=c_{1} a_{n-1}+c_{2} a_{n-2}+\cdots+c_{n-1} a_{1}+n c_{n} .
$$

Du, Huang and Li showed that the classes of Newton sequences generated by integers and Dold sequences in fact coincide [10]. As a result, due to the fact that each integer valued generating sequence produce a Newton sequence, we obtain a new description of Dold sequences.

In number-theoretical considerations Dold sequences play important role: many classical sequences turned out to belong to this class, furthermore various techniques (for example using generating sequences) applied to Dold sequences enable to obtain new types of congruences $[10,11]$.

The aim of this paper is to generalize the notion of Dold sequences for the case of partially ordered sets (posets). We provide an overall look at the topic, presenting the construction in which classical Dold sequences are the special case with the order given by relation of divisibility. The poset approach seems to be a natural bridge joining the topological notion of Dold sequences with combinatorics. We should stress here that the concept of the Möbius function on poset appeared more then 50 years ago (see paper [33] by Rota, and Stanley's book [38]). It has been intensively investigated since then (see e.g. effective applications of Möbius technics on posets to solve interesting combinatorial questions $[3,5,34,37,39]$, with probably the latest one [2]). In the present paper we refer 
to the Möbius function as a concept that naturally defines the Dold sequences on posets and enables us to give its combinatorial interpretation in terms of counting the periodic points.

The paper is organized in the following way. In the second section we give the definition and examples of standard Dold sequences as well as describe their basic properties. In the next section we introduce generalized Dold sequences. Here we also prove that, analogously to classical case, every such sequence has a representation as the combination of some elementary sequences, denoted as $\operatorname{Reg}_{k}$ (Theorem 16). Section 4 is devoted to generalization of the result of Wójcik in [43], who provided the equivalent definition of Dold sequences by arithmetical functions different from the Möbius function. We describe such characterization for generalized arithmetical function, i.e. functions defined on a partially ordered set (Theorem 22). In Section 5 we consider some special case of the theory and analyze Dold vector sequences as well as Dold matrix sequences. We provide a combinatorial model within periodic point theory, in which vector Dold sequences may be interpreted as the sequences of fixed points of a given type, and the order is given by inclusion (Theorem 27). The same construction may be also performed for fixed points classified according to two independent features (Remark 36).

\section{Classical Dold sequences}

In this section we introduce the classical Dold sequences for the order given by relation of divisibility.

Through the whole paper we apply the following terminology: $x$ is a periodic point with minimal period $n$ if $f^{n}(x)=x$ and $f^{m}(x) \neq x$ for $m<n$ (we will also call $x$ an $n$-periodic point for short). The orbit of such a point will be called $n$-orbit, or an orbit of lenght $n$.

Definition 1. A sequence of integers $\left(a_{n}\right)_{n=1}^{\infty}$ is called a Dold sequence if the following congruences (called Dold congruences or Dold relations) are fulfilled:

$$
\sum_{k \mid n} \mu\left(\frac{n}{k}\right) a_{k} \equiv 0(\bmod n) \text { for each } n \geqslant 1,
$$

where $\mu: \mathbb{N} \rightarrow \mathbb{Z}$ is the classical Möbius function, given by the formula

$$
\mu(n)=\left\{\begin{array}{l}
1, \text { if } n=1 \\
(-1)^{k}, \text { if } n=p_{1} p_{2} \ldots p_{k} \text { for different primes } p_{i} \\
0, \text { otherwise. }
\end{array}\right.
$$

There is a convenient way of representing a Dold sequence by a use of so-called periodic expansion, i.e. as a combination of some basic periodic sequences, with some integer coefficients called Dold coefficients. 
Definition 2. Let $k$ be a fixed natural number. We define

$$
\operatorname{reg}_{k}(n)= \begin{cases}k & \text { if } k \mid n, \\ 0 & \text { if } k \nmid n\end{cases}
$$

Thus, reg $_{k}$ is the periodic sequence:

$$
\left(\operatorname{reg}_{k}(n)\right)_{n \geqslant 1}=(0, \ldots, 0, k, 0, \ldots, 0, k, \ldots),
$$

where the non-zero entries appear for indices divisible by $k$.

Proposition 3. (cf. [24] Proposition (3.2.7)) Any arithmetic function $\left(g_{n}\right)_{n=1}^{\infty}$ can be written uniquely in the following form of a periodic expansion:

$$
g_{n}=\sum_{k=1}^{\infty} \alpha_{k} \operatorname{reg}_{k}(n), \text { where } \alpha_{n}=\frac{1}{n} \sum_{k \mid n} \mu\left(\frac{n}{k}\right) g(k) \in \mathbb{Q} .
$$

Moreover, $g$ is integer valued and satisfies Dold congruences iff $\alpha_{k} \in \mathbb{Z}$ for every $k \in \mathbb{N}$.

Remark 4. By Proposition 3 every Dold sequence is an integer combination (possibly infinite) of basic periodic sequences reg . .

Definition 5. For a given Dold sequence $\left(a_{n}\right)_{n}$ we define $\left(b_{n}\right)_{n}$, the sequence of Dold coefficients, by the formula:

$$
b_{n}=\frac{1}{n} \sum_{k \mid n} \mu\left(\frac{n}{k}\right) a_{k}
$$

Remark 6. Notice that by Proposition 3 the sequence of Dold coefficients is an integer sequence. Furthermore by the application of the Möbius Inversion Formula (cf. [38] Section 3.7) for a given $\left(b_{n}\right)_{n}$, one may uniquely determine the sequence $\left(a_{n}\right)_{n}$, namely:

$$
a_{n}=\sum_{k \mid n} k b_{k}
$$

For $k, l \in \mathbb{N}$ let us denote by $(k, l)$ the greatest common divisor and by $[k, l]$ the least common multiple of $k$ and $l$. The following multiplication rule for basic sequences holds (cf. also [24]):

$$
\operatorname{reg}_{k} \cdot \operatorname{reg}_{l}=(k, l) \operatorname{reg}_{[k, l]} \cdot
$$

Corollary 7. The product and the sum of Dold sequences is a Dold sequence. As a consequence, they constitute the subring of integer-valued sequences.

Proof. Indeed, if $(a)_{n}$ and $\left(b_{n}\right)_{n}$ are Dold sequences then each of them is a combination of basic sequences. Thus (obviously) $\left(a_{n}+b_{n}\right)_{n}$ and $\left(a_{n} \cdot b_{n}\right)_{n}$ by the formula (6) are also some combination of basic sequences. 
Below, we will give some examples of Dold sequences.

Example 8. (1) Let $X$ be a set and let $f: X \rightarrow X$. Denote by \# $\operatorname{Fix}\left(f^{n}\right)$ the number of fixed points of the $n$-th iterate $f^{n}$ and assume that it is finite. Then the sequence $\left(a_{n}\right)_{n}, a_{n}=\# \operatorname{Fix}\left(f^{n}\right)$ is a Dold sequence. This results from the fact that each Dold coeficient $b_{n}$ (defined in (4)) is equal to the number of $n$-periodic points, i.e. is the product of $n$ and the number of $n$-orbits (cf. [12]).

(2) Let $A$ be a square matrix with integer values. The sequence given by the formula $a_{n}=\operatorname{tr} A^{n}$ is a Dold sequence (cf. [40] and the references therein).

(3) Let $Y$ be an Euclidean Neighbourhood Retract (ENR) and $V \subset Y$ be an open subset. Consider $f: V \rightarrow Y$, a continuous map, and the iterations $f^{n}: V_{n} \rightarrow Y$, where $V_{n}$ is defined inductively by $V_{1}=V, V_{n}=f^{-1}\left(V_{n-1}\right)$ for $n>1$. Under the assumption that $\operatorname{Fix}\left(f^{n}\right)$ is compact, the sequence of fixed point indices $\left(\operatorname{ind}\left(f^{n}, V_{n}\right)\right)_{n}$ is a Dold sequence [8].

(4) There is a huge collection of well-known sequences that appear in combinatorics, for example Lucas sequences, that are Dold sequences, see the examples given in [31] from The On-Line Encyclopedia of Integer Sequences [29].

Remark 9. Dold sequences of the type (1) of Example 8 provide a lot of important congruences - for example taking the map $f: \mathbb{C} \rightarrow \mathbb{C}, f(z)=z^{a}, a \in \mathbb{N}, a \geqslant 2$ we get that $a_{n}=\# \operatorname{Fix}\left(f^{n}\right)=a^{n}$ defines a Dold sequence. Thus, taking $n=p$ a prime, we obtain $a^{p}-a \equiv 0(\bmod p)$, i.e classical Little Fermat Theorem. More elaborated maps produce more complicated congruences.

By (2) in Example 8 we get that the sequence of Lefschetz numbers of iterations $\left(L\left(f^{n}\right)\right)_{n}$ is a Dold sequences, as it is equal to $\operatorname{tr} A^{n}-\operatorname{tr} B^{n}$ for some integer valued square matrices $A$ and $B$. Let us notice here, that Lefschetz numbers of iterations are very useful device in periodic point theory, cf. $[1,4,9,18,23,36]$ and the references therein.

Dold sequences of the type (3) provide restrictions for sequences of local fixed point indices (at a fixed point $x_{0}$ ) (ind $\left.\left(f^{n}, x_{0}\right)\right)_{n}$ for various classes of maps $f$, which is important from the point of view of study of their dynamics and the structure of periodic points.

Dold sequences are strictly related to periodic point theory by the notion of exactly realizable sequences.

Definition 10. A sequence $\left(a_{n}\right)_{n}$ with values in $\mathbb{N} \cup\{0\}$ is called exactly realizable if there is a space $X$ and a map $f: X \rightarrow X$ such that $a_{n}=\# \operatorname{Fix}\left(f^{n}\right)$ for all $n \geqslant 1$.

A full characterization of exactly realizable sequences by Dold sequences is given by the following statement.

Lemma 11. (cf. [31]) Let $\left(a_{n}\right)_{n}$ be a sequence of non-negative integers. Then $\left(a_{n}\right)_{n}$ is exactly realizable if and only if $\left(a_{n}\right)_{n}$ is a Dold sequence and all Dold coefficients $b_{n}$ (given in Definition 5) are non-negative. 
Recently, Wójcik in [43] has made an interesting observation that the Möbius function $\mu$ in Definition 1 may be replaced by any other integer valued function satisfying some natural conditions. In this way a new description of Dold sequences is obtained.

Theorem 12. [43] Let $\psi: \mathbb{N} \rightarrow \mathbb{Z}$ be a function satisfying the following conditions:

$$
\sum_{k \mid n} \psi(k) \equiv 0(\bmod n) ; \quad \psi(1)= \pm 1
$$

Then a sequence $\left(a_{n}\right)_{n}$ is a Dold sequence if and only if

$$
\sum_{k \mid n} \psi\left(\frac{n}{k}\right) a_{k} \equiv 0(\bmod n) \text { for each } n \geqslant 1
$$

Notice that the condition (7) is obviously satisfied by the Möbius function, as the considered sum is equal to zero. It is also satisfied for much larger class of maps, for example for the Euler function $\phi$ (that counts the positive integers up to a given integer $n$ that are relatively prime to $n)$. Indeed, $\sum_{k \mid n} \phi(k)=n \equiv 0(\bmod n)$.

\section{Generalized Dold sequences and their properties}

Let $P$ be a countable partially ordered set (poset) with a partial order " $\leqslant$ " and $w: P \rightarrow \mathbb{Z}$ be a function assigning to each $x \in P$ a weight $w \in \mathbb{Z}$.

We will assume that the following conditions are satisfied:

(A1) for each $p \in P$ the set $\{q: q \leqslant p\}$ is finite,

(A2) $\forall_{x, y \in P} x<y \Rightarrow w(x) \mid w(y) \wedge w(x)<w(y)$.

Such a poset may be identified with a directed graph (this graph is often called the Hasse diagram). Then the elements of the poset correspond to vertices and a directed edge from $x \in P$ to $y \in P$ exists iff $x<y$ and there is no $z \in P \backslash\{x, y\}$ such that $x \leqslant z \leqslant y$.

In such a graph a generalized Möbius function $\mu^{*}: P \times P \rightarrow \mathbb{Z}$ is defined by the formula:

$$
\mu^{*}(x, y)=\left\{\begin{array}{l}
1 \text { if } x=y, \\
-\sum_{x \leqslant z<y} \mu^{*}(x, z) \text { if } x<y, \\
0 \text { otherwise. }
\end{array}\right.
$$

We define also two other important functions: $\zeta, \delta: P \times P \rightarrow \mathbb{R}$.

$$
\begin{aligned}
& \delta(x, y)=\left\{\begin{array}{l}
1 \text { if } x=y \\
0 \text { otherwise }
\end{array}\right. \\
& \zeta(x, y)=\left\{\begin{array}{l}
1 \text { if } x \leqslant y \\
0 \text { otherwise }
\end{array}\right.
\end{aligned}
$$


Now we are in position to define generalized Dold sequence (assuming $P$ satisfies the conditions (A1) and (A2)).

Definition 13. A sequence $\left(a_{p}\right)_{p \in P}$ indexed by the elements of $P$ satisfies Dold relations with respect to weights $w: P \rightarrow \mathbb{Z}$ if for each $y \in P$

$$
\sum_{x \leqslant y} \mu^{*}(x, y) a_{x} \equiv 0(\bmod w(y))
$$

If $\left(a_{p}\right)_{p \in P}$ satisfies Definition 13 we will also say that it is a generalized Dold sequence.

\subsection{Representation of generalized Dold sequence as a combination of basic periodic sequences}

For any two elements $x, y \in P$ such that $x \leqslant y$ we may define the interval

$$
\operatorname{Int}[x, y]=\{z \in P: x \leqslant z \leqslant y\} .
$$

We call a poset $P$ locally finite if each interval is finite. Note that by the assumption (A1) we consider only locally finite posets.

We consider the so-called incidence algebra (denoted by $\mathcal{A}$ ). It is $\mathbb{R}$-algebra over the vector space of real-valued functions defined on such intervals (satisfying the condition: $f(x, y)=0$ for all such $x, y \in P$ that $\neg(x \leqslant y)$ ), equipped with the bilinear product called convolution, denoted by $*$, and given by the formula:

$$
(f * g)(x, y)=\sum_{z: x \leqslant z \leqslant y} f(x, z) g(z, y) .
$$

We will interpret $\mathcal{A}$ as the ring with unity $\delta$. Then the inverse element to $\mu^{*}$ is $\zeta$ :

$$
\zeta * \mu^{*}=\mu^{*} * \zeta=\delta .
$$

In this case, the following theorem (known as the Möbius inversion formula) holds (cf. [38]):

Theorem 14. Let $b$ be a real-valued sequence defined on $P$. Then the equality

$$
S_{b}(x):=\sum_{y: y \leqslant x} b(y)
$$

is equivalent to:

$$
b(x)=\sum_{y: y \leqslant x} \mu^{*}(y, x) S_{b}(y) .
$$

Proof. We add to the order $P$ the smallest element $m$ and assume that $b(m)=0$. Next, we define the function $f \in \mathcal{A}$ as:

$$
f(x, y)=\left\{\begin{array}{l}
b(y) \text { if } x=m \\
0 \text { otherwise }
\end{array}\right.
$$


Let $g=f * \zeta$, then:

$$
g(m, y)=\sum_{z: m \leqslant z \leqslant y} f(m, z) \zeta(z, y)=\sum_{z: z \leqslant y} b(z)=S_{b}(y) .
$$

By the equality $f=g * \zeta^{-1}=g * \mu^{*}$ we get

$$
b(x)=f(m, x)=\sum_{y: m \leqslant y \leqslant x} g(m, y) \mu^{*}(y, x)=\sum_{y: m \leqslant y \leqslant x} S_{b}(y) \mu^{*}(y, x) .
$$

Now we define the counterpart of the sequences reg $_{k}$ and show that every generalized Dold sequence may be represented uniquely as the combination of such sequences, which gives a generalization of Proposition 3.

Definition 15. For each element $p \in P$ let $\operatorname{Reg}_{p}: P \rightarrow \mathbb{Z}$ be given by the formula

$$
\operatorname{Reg}_{p}(x)=\left\{\begin{array}{l}
w(p) \text { if } p \leqslant x \\
0 \text { otherwise }
\end{array}\right.
$$

Theorem 16. A sequence $(a(x))_{x \in P}$ satisfies Dold relations with respect to weights $w$ if and only if there is uniquely determined sequence of integers $\left(b_{x}\right)_{x \in P}$ such that

$$
a(x)=\sum_{y \leqslant x} b_{y} \operatorname{Reg}_{y}(x) .
$$

Proof. By Theorem 14 the equality $a(x)=\sum_{y \leqslant x} b_{y} \operatorname{Reg}_{y}(x)$ is equivalent to

$$
b_{x} \operatorname{Reg}_{x}(x)=\sum_{y \leqslant x} \mu^{*}(y, x) a(y) .
$$

Taking into account that the left-hand side of the equality (19) is equal either 0 or $b_{x} \cdot w(x)$ we get that: if all $b_{x}$ are integers, then $(a(x))_{x \in P}$ is a generalized Dold sequence. Conversely, if $(a(x))_{x \in P}$ is a generalized Dold sequence then all $b_{x}$ are integers.

Remark 17. Notice that if $a(x)=S_{c}(x)=\sum_{y \leqslant x} c(y)$, then by (19) $\left(a_{x}\right)_{x \in P}$ is a generalized Dold sequence if and only if $w(x) \mid c(x)$ for each $x$.

If sequences $\left(a_{p}\right)_{p \in P},\left(a_{p}^{\prime}\right)_{p \in P}$ satisfy Dold relations with respect to weights $w$, then obviously their combination with integer coefficients also satisfies the relations. However, the similar property concerning the product of two sequences satisfying Dold relations does not hold, which can be seen by the following example.

Example 18. Let $P=\{A, B, C\}$ and $A<C$, and $B<C$, and neither $A<B$, nor $B<A$. We define the weights: $w(A)=w(B)=1, w(C)=2$.

Then the condition (A2) is satisfied and $\mu^{*}(A, A)=\mu^{*}(B, B)=\mu^{*}(C, C)=1, \mu^{*}(A, C)=$ $\mu^{*}(B, C)=-1, \mu^{*}(A, B)=\mu^{*}(B, A)=\mu^{*}(C, A)=\mu^{*}(C, B)=0$. 
If a sequence $\left(a_{x}\right)_{x \in P}$ satisfies (12), then the only non-trivial relation is:

$$
a_{C}-a_{B}-a_{A} \equiv 0(\bmod 2) .
$$

Consider the sequences $\left(a_{x}\right)_{x \in P},\left(a_{x}^{\prime}\right)_{x \in P}$ satifying (20). Then they are of the form:

$a_{A}=m, a_{B}=n, a_{C}=2 k+m+n, k, m, n \in \mathbb{Z}$ and $a_{A}^{\prime}=p, a_{B}^{\prime}=r, a_{C}^{\prime}=2 s+p+r$, $p, r, s \in \mathbb{Z}$. Then the sequence $\left(a_{x} \cdot a_{x}^{\prime}\right)_{x \in P}$ does not satisfy (20) if $m r+n p$ is odd.

An interesting class of sequences that turned out to be Dold sequences were considered in $[35]$.

Definition 19. A sequence $a: \mathbb{N} \times \mathbb{N} \rightarrow \mathbb{R}$ is called a two-dimensional Dold sequence if for any natural numbers $m$ and $n$,

$$
\sum_{k \mid n} \sum_{l \mid m} \mu\left(\frac{n}{k}\right) \mu\left(\frac{m}{l}\right) a(k, l) \equiv 0(\bmod [m, n]) .
$$

To reveal the structure of two-dimensional Dold sequences, we first cite the following lemma cf. ([38]).

Lemma 20. Let $P_{1}, P_{2}$ be two locally finite posets equipped with the relation $\leqslant P_{1} \times P_{2}$, where $(x, y) \leqslant_{P_{1} \times P_{2}}(z, w) \Longleftrightarrow x \leqslant_{P_{1}} z$ and $y \leqslant_{P_{2}} w$. Then $\mu_{P_{1} \times P_{2}}((x, y),(z, w))=$ $\mu_{P_{1}}(x, z) \cdot \mu_{P_{2}}(y, w)$.

Theorem 21. Every two-dimensional Dold sequence is a generalized Dold sequence.

Proof. Notice that for $P=\mathbb{N}$ and the order given by the divisibility relation, we have for all natural $k, n$ :

$$
\mu^{*}(k, n)=\mu(n / k) .
$$

We define the relation in $P \times P=\mathbb{N} \times \mathbb{N}$ in the following way: $\left(k_{1}, l_{1}\right) \leqslant\left(k_{2}, l_{2}\right) \Longleftrightarrow$ $k_{1}\left|k_{2} \wedge l_{1}\right| l_{2}$ and take the weights: $w(k, l)=[k, l]$.

By Lemma 20 and the equality $(21) \mu_{P \times P}^{*}((k, l)(n, m))=\mu^{*}(k, n) \cdot \mu^{*}(l, m)=\mu(n / k)$. $\mu(m / l)$.

Finally,

$$
\sum_{k \mid n} \sum_{l \mid m} \mu\left(\frac{n}{k}\right) \mu\left(\frac{m}{l}\right) a(k, l) \equiv 0(\bmod [m, n])
$$

is equivalent to:

$$
\sum_{(k, l) \leqslant(n, m)} \mu^{*}((k, l),(n, m)) \cdot a(k, l) \equiv 0(\bmod w(m, n)) .
$$




\section{Characterization of generalized Dold sequences by a use of various generalized arithmetical functions}

In this section we prove that the generalized Möbius function in the conditions (12) may be replaced by some other functions. Our result generalizes the main theorem of Wójcik in [43] i.e. Theorem 12.

For a given map $\varphi: P \times P \rightarrow \mathbb{Z}$ we define the map $\tilde{S}_{\varphi}: P \times P \rightarrow \mathbb{Z}$ by the formula $\tilde{S}_{\varphi}(z, x)=\sum_{y: z \leqslant y \leqslant x} \varphi(y, x)$.

Theorem 22. Assume that $\varphi(x, x)= \pm 1$ and $\tilde{S}_{\varphi}(y, x) \equiv 0\left(\bmod \frac{w(x)}{w(y)}\right)$ for all $x, y$. Then $\left(S_{b}(x)\right)_{x \in P}$ is a generalized Dold sequence if and only if $\sum_{y: y \leqslant x} \varphi(y, x) S_{b}(y) \equiv 0(\bmod w(x))$ for each $x \in P$.

Proof. In the following proof we assume that $b(x)$ is given by (15). First we will prove

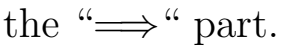

Because $S_{b}$ is a Dold sequence thus, by Remark 17, $w(z) \mid b(z)$ for each $z$, i.e. there is $d_{z} \in \mathbb{Z}$ such that $b(z)=w(z) \cdot d_{z}$.

The assumption $\tilde{S}_{\varphi}(y, x) \equiv 0\left(\bmod \frac{w(x)}{w(y)}\right)$ implies that for all $z \leqslant x$ there exists $k_{(z, x)} \in \mathbb{Z}$ such that $\tilde{S}_{\varphi}(z, x)=\frac{w(x)}{w(z)} \cdot k_{(z, x)}$. Then we have:

$$
\begin{aligned}
& \sum_{y: y \leqslant x} \varphi(y, x) S_{b}(y)=\sum_{y: y \leqslant x} \varphi(y, x) \sum_{z: z \leqslant y} b(z)=\sum_{z: z \leqslant x} b(z) \sum_{y: z \leqslant y \leqslant x} \varphi(y, x) \\
= & \sum_{z: z \leqslant x} b(z) \tilde{S}_{\varphi}(z, x)=\sum_{z: z \leqslant x} w(z) \cdot d_{z} \cdot \frac{w(x)}{w(z)} \cdot k_{(z, x)}=w(x) \sum_{z: z \leqslant x} d_{z} k_{(z, x)} .
\end{aligned}
$$

Therefore $\sum_{y: y \leqslant x} \varphi(y, x) S_{b}(y) \equiv 0(\bmod (w(x))$.

Now, using the induction, we will prove the opposite implication " $\Longleftarrow "$. i.e. that $\sum_{y: y \leqslant x} \varphi(y, x) S_{b}(y) \equiv 0(\bmod w(x)) \Longrightarrow S_{b}$ is a Dold sequence, which by Remark 17 is equivalent to the condition that

$$
w(x) \mid b(x) .
$$

If $x$ is a minimal element in $P$, then

$$
\sum_{y: y \leqslant x} \varphi(y, x) S_{b}(y)=\varphi(x, x) S_{b}(x)=\varphi(x, x) b(x)= \pm b(x) \equiv 0(\bmod w(x)) .
$$

Therefore our condition (23) is satisfied.

Let us take $x \in P$ and make the inductive assumption that for all $z<x$ we have $w(z) \mid b(z)$. Assume now that $\sum_{y: y \leqslant z} \varphi(y, z) S_{b}(y) \equiv 0(\bmod w(z))$. Then, using in the first 
equality below the same rearrangement as in (22) we get:

$$
\begin{aligned}
\sum_{y: y \leqslant x} \varphi(y, x) S_{b}(y) & =\sum_{z: z \leqslant x} b(z) \tilde{S}_{\varphi}(z, x)=\sum_{z: z<x} b(z) k_{(z, x)} \frac{w(x)}{w(z)}+b(x) \tilde{S}_{\varphi}(x, x) \\
& =\sum_{z: z<x} \frac{b(z)}{w(z)} k_{(z, x)} w(x)+b(x) \varphi(x, x) .
\end{aligned}
$$

By the inductive assumption $\sum_{z: z<x} b(z) \tilde{S}_{\varphi}(z, x) \equiv 0(\bmod w(x))$, thus, taking into account that $\phi(x, x)= \pm 1$ we obtain the congruence $(23)$.

\section{Vector Dold sequences and their interpretation}

\subsection{Generalized Dold sequences and periodic points}

Now we add an additional assumption (A3) to (A1) and (A2) for a considered poset $P$ having a relation " $\leqslant$ " with weights.

(A3) Let $N \in \mathbb{N}$ be fixed. For each weight $k \in \mathbb{N}$ there exists at most (we may assume that exactly) $N$ elements of $P$ with the weight $k$.

So, since now, we assume that elements of the poset $P$ are given as pairs $(k, i)$ where $k \in\{1,2, \ldots\}$ and $i \in\{1, \ldots, N\}$. To simplify notation instead of writing $(k, i)$ we will write $k_{i}$ i.e. we will denote the points in the poset $P$ with the weight $k$ as $k_{1}, \ldots, k_{N}$ (there are $N$ copies of $k$ ). In this circumstances the assumption (A2) takes the form

$$
k_{i} \leqslant l_{j} \Rightarrow k \mid l \text {. }
$$

The above condition is the necessary one for the relation to hold, but in order to describe the relation completely we introduce certain matrix notation. Below, for a matrix $A$, by $A(i, j)$ we denote its entry lying in $i$-th row and $j$-th column.

Let us assume that for any natural numbers $k, l \in \mathbb{N}$ we have the matrix $S_{k \rightarrow l}$ such that

$$
S_{k \rightarrow l}(b, a)= \begin{cases}1 & \text { if } k_{a} \leqslant l_{b}, \\ 0 & \text { if } k_{a} \nless l_{b} .\end{cases}
$$

So in $b$-th row and $a$-th column of the matrix $S_{k \rightarrow l}$ there is 1 or 0 depending whether $k_{a}$ is related to $l_{b}$ or not. We call these matrices characteristic matrices of the relation $\leqslant$. Note that $S_{k \rightarrow k}$ is the identity matrix for any $k \in \mathbb{N}$.

We will now consider the certain model of the partial order relation related to periodic points theory, in which the order reflects how the shorter orbits are assigned to longer orbits, i.e. defines the filtrations of the sets of orbits in respect to their length.

Remark 23. Let $g: X \rightarrow X$ be a (continuous) map such that for each $k$ the set $\operatorname{Fix}\left(g^{k}\right)$ is finite. We assume that all $k$-orbits of the map $g$ are classified into finite set of types, 
where types correspond to the numbers from the finite set $\{1, \ldots, N\}$. In this way, the set of orbits for each $k$ is divided into (not necessarily disjoint) families $\left\{k_{1}, \ldots, k_{N}\right\}$, where $k_{i}$ denotes orbits of the length $k$ belonging to the type $i$.

We will say that a fixed point $x_{0} \in \operatorname{Fix}\left(g^{n}\right)$ has the target type $i(n)$ if it belongs to an $k$-orbit in $k_{j}$ such that $k_{j} \leqslant n_{i}$. Note that there is no limitation in the number of target types a fixed point is assigned to. The set of all fixed points of $g^{n}$, having the target type $i$, will be denoted as $\operatorname{Fix}_{i}\left(g^{n}\right)$.

In this context we have the natural definition of the vector valued sequence $X_{n} \in \mathbb{R}^{N}$, $n \in \mathbb{N}$ given by

$$
X_{n}(i)=\# \operatorname{Fix}_{i}\left(g^{n}\right) .
$$

Let $A_{k} \in \mathbb{R}^{N}$ be the vector whose $j$-th coordinate $A_{k}(j)$ equals to the number of orbits of length $k$ and belonging to the type $j$. Then the formula (26) for $X_{n}(i)$ takes the form:

$$
X_{n}(i)=\sum_{k: k_{j} \leqslant n_{i}} k \cdot A_{k}(j)
$$

Since the points $k_{i}$ and $l_{j}$ are joined (cf. (25)) if and only if $S_{k \rightarrow l}(j, i)=1$, and $S_{k \rightarrow l}$ is $0-1$ matrix, the following equality holds:

$$
X_{n}=\sum_{k: k \mid n} k S_{k \rightarrow n} A_{k}=\sum_{k=1}^{n} k S_{k \rightarrow n} A_{k},
$$

the last equality being valid since we may assume that if $k \not 1 n$, then $S_{k \rightarrow n}=0$. This is a natural generalization of the classical situation when we count all periodic points, without dividing them into types and have one dimensional situation of

$$
x_{n}=\sum_{k: k \mid n} k a_{k}
$$

where $a_{k}$ denotes the number of periodic $k$-orbits and $x_{n}=\#$ Fix $g^{n}$. In this one dimensional interpretation $S_{k \rightarrow n}$ is a $1 \times 1$ matrix given by

$$
S_{k \rightarrow n}= \begin{cases}1 & \text { for } k \mid n \\ 0, & \text { for } k \not h .\end{cases}
$$

Below we provide some examples of classifying orbits into types.

Example 24. Let us fix the set $U \subset X$ and for each orbit $A$ define the type of the orbit $A$ as

$$
t(A)= \begin{cases}2 & \text { if } A \cap U \neq \emptyset, \\ 1 & \text { if } A \cap U=\emptyset .\end{cases}
$$

Thus, $t(A)=2$ if and only if the orbit $A$,visits" the set $U$. As a consequence, we have $N=2$ types and the relation is defined as 


$$
k_{i}<n_{j} \Leftrightarrow k \mid n \wedge i \leqslant j \wedge k<n .
$$

This means that when counting fixed points of $g^{n}$ of the type 1 we count only these orbits that have not visited the set $U$, while the type 2 covers all orbits.

Example 25. The previous setting may be extended and modified to more complex situation. Let $\sigma: X \rightarrow \mathbb{R}$ be any function taking values in the finite set $\{1, \ldots, N\}$ and for each finite orbit $A$ of the map $g: X \rightarrow X$ the type of the orbit $A$ is given by

$$
t(A)=\max \{\sigma(x): x \in A\} .
$$

Then the elements of the poset $P$ are given as pairs $k_{i} \in \mathbb{N} \times\{1, \ldots, N\}$ where $k_{i}$ is identified with the set of $k$-orbits $A$ having the same type $t(A)=i$. Of course for each $k$ there exists only finite number of non-empty sets of orbits described by pairs $k_{i}$. The relation is given by

$$
k_{i} \leqslant n_{j} \Longleftrightarrow k \mid n \wedge i=j .
$$

\subsection{The Möbius matrix function}

Now we define the Möbius matrix function by

$$
M_{k \rightarrow l}^{*}=\left[\mu^{*}\left(k_{i}, l_{j}\right)\right]_{1 \leqslant j, i \leqslant N}
$$

for $k, l \in \mathbb{N}$. We use the convention that $M_{k \rightarrow l}^{*}(j, i)=\mu^{*}\left(k_{i}, l_{j}\right)$ so in $j$-th row and $i$-th column is the generalized Möbius function for the pair of points $k_{i}$ and $l_{j}$.

We are going to show that the Möbius matrix function satisfies some recurrence relations similar to what is known from the classical case. Let us start from the recurrence relation for the Möbius function $\mu^{*}$ defined on poset, which is an equivalent of the formula (9) applied under the condition (A3).

$$
\mu^{*}\left(k_{i}, l_{j}\right)=\left\{\begin{array}{l}
1 \text { if } k_{i}=l_{j}, \\
-\sum_{n_{c}: k_{i} \leqslant n_{c}<l_{j}} \mu^{*}\left(k_{i}, n_{c}\right) \text { if } k_{i}<l_{j}, \\
0 \text { otherwise }
\end{array}\right.
$$

Note that:

$$
M_{k \rightarrow k}^{*}=I
$$

where $I$ denotes the identity matrix.

Moreover, for $l>k$ we have

$$
M_{k \rightarrow l}^{*}(j, i)=\mu^{*}\left(k_{i}, l_{j}\right)=-\sum_{n: k|n \wedge n| l \wedge n \neq l} \sum_{c=1}^{N} S_{k \rightarrow n}(c, i) S_{n \rightarrow l}(j, c) \mu^{*}\left(k_{i}, n_{c}\right) .
$$


Note however, that if $S_{k \rightarrow n}(c, i)=0$ then also $\mu^{*}\left(k_{i}, n_{c}\right)=0$, so we may actually replace $S_{k \rightarrow n}(c, i)$ with 1 without changing the value of the sum (31). Hence we have

$$
\mu^{*}\left(k_{i}, l_{j}\right)=-\sum_{n: k|n \wedge n| l \wedge n \neq l} \sum_{c=1}^{N} S_{n \rightarrow l}(j, c) \mu^{*}\left(k_{i}, n_{c}\right) .
$$

Let us remind that $\mu^{*}\left(k_{i}, n_{c}\right)=M_{k \rightarrow n}^{*}(c, i)$ thus the sum $\sum_{c=1}^{N} S_{n \rightarrow l}(j, c) \mu^{*}\left(k_{i}, n_{c}\right)$ corresponds to the multiplication of $j$-th row of $S_{n \rightarrow l}$ and $i$-th column of $M_{k \rightarrow n}^{*}$. Hence we can write in the matrix notation:

$$
M_{k \rightarrow l}^{*}=-\sum_{n: k|n \wedge n| l \wedge n \neq l} S_{n \rightarrow l} M_{k \rightarrow n}^{*} .
$$

We are ready to introduce the notion of a vector Dold sequence.

Definition 26. A sequence of vectors $\left(X_{n}\right)_{n \in \mathbb{N}} \in \mathbb{Z}^{N}$ is a vector Dold sequence if for each $n \in \mathbb{N}$ there exists integer valued vector $J_{n} \in \mathbb{Z}^{N}$ such that

$$
\sum_{k \mid n} M_{k \rightarrow n}^{*} X_{k}=n J_{n}
$$

Now we will show that our model based on the classification of periodic orbits, described in Remark 23, produces in a natural way a vector Dold sequence.

Theorem 27. The vector sequence $\left(X_{n}\right)_{n}$ given by the equality (28) is a vector Dold sequence.

Proof. Assume now the integer vector valued sequences $A_{n}$ and $X_{n}$ are joined by the relation $(27)$, i.e.

$$
X_{n}(i)=\sum_{k_{j} \leqslant n_{i}} k A_{k}(j)
$$

By Theorem 14 with $S_{a}\left(k_{j}\right)=X_{k}(j)$ and $a\left(k_{j}\right)=k A_{k}(j)$ we obtain:

$$
n A_{n}(i)=\sum_{k_{j} \leqslant n_{i}} \mu^{*}\left(k_{j}, n_{i}\right) X_{k}(j)
$$

Looking at the formula above for the entire vectors $A_{n}$ and $X_{k}$, we have

$$
n A_{n}=\sum_{k \mid n} M_{k \rightarrow n}^{*} X_{k}
$$

which proves that $X_{n}$ is a vector Dold sequence. 


\subsection{Representation of vector and matrix Dold sequences by Reg function.}

Let us define the matrix function $\mathrm{REG}_{k}: \mathbb{N} \rightarrow \operatorname{Mat}_{N \times N}(\mathbb{Z})$ given by

$$
\operatorname{REG}_{k}(n)=k \cdot S_{k \rightarrow n}
$$

for $k \in \mathbb{N}$. By the definition of the matrix $S_{k \rightarrow n}, k \not h$ implies $\operatorname{REG}_{k}(n)=0$.

Theorem 28. The sequence of vectors $\left(X_{n}\right)_{n \in \mathbb{N}} \in \mathbb{Z}^{N}$ is a vector Dold sequence if and only if there exists a sequence of integer vectors $\left(B_{n}\right)_{n \in \mathbb{N}} \in \mathbb{Z}^{N}$ such that

$$
X_{n}=\sum_{k \mid n} \operatorname{REG}_{k}(n) B_{k}
$$

for each $n \in \mathbb{N}$.

Proof. Assume that $\left(X_{n}\right)_{n \in \mathbb{N}} \in \mathbb{Z}^{N}$ is a vector Dold sequence. Hence $k_{i} \mapsto a\left(k_{i}\right)=X_{k}(i)$ is a Dold sequence for the poset $P$ defined on pairs $k_{i}$, where $(k, i) \in \mathbb{N} \times\{1, \ldots, N\}$ with the order relation defined by matrices $S_{k \rightarrow n}$.

By Theorem 16 the sequence $\left(a\left(n_{i}\right)\right)_{n_{i} \in P}$ is a Dold sequence on $P$ iff there exists a sequence $\left(b\left(n_{i}\right)\right)_{n_{i} \in P}$ such that

$$
a\left(n_{j}\right)=\sum_{k_{i} \leqslant n_{j}} b\left(k_{i}\right) \operatorname{Reg}_{k_{i}}\left(n_{j}\right) .
$$

Let us fix $j \in\{1, \ldots, N\}$. Then

$$
X_{n}(j)=a\left(n_{j}\right)=\sum_{k_{i} \leqslant n_{j}} b\left(k_{i}\right) \operatorname{Reg}_{k_{i}}\left(n_{j}\right)=\sum_{k \mid n} \sum_{i=1}^{N} b\left(k_{i}\right) \operatorname{Reg}_{k_{i}}\left(n_{j}\right),
$$

since if $k \not n$ then $\operatorname{Reg}_{k_{i}}\left(n_{j}\right)=0$, then

$$
\operatorname{Reg}_{k_{i}}\left(n_{j}\right)=k \Leftrightarrow S_{k \rightarrow n}(j, i)=1 .
$$

Thus, continuing (38) we obtain, for a fixed $j \in\{1, \cdots, N\}$ :

$$
\sum_{k \mid n} \sum_{i=1}^{N} b\left(k_{i}\right) \operatorname{Reg}_{k_{i}}\left(n_{j}\right)=\sum_{k \mid n} \sum_{i=1}^{N} k S_{k \rightarrow n}(j, i) b\left(k_{i}\right)=\sum_{k \mid n}\left(\operatorname{REG}_{k}(n) B_{k}\right)_{j},
$$

where $(X)_{j}$ means $j$-th coordinate of the vector $X$. Since the above reasoning may be repeated for any $j \in\{1, \ldots, N\}$ we obtain that

$$
X_{n}=\sum_{k \mid n} \operatorname{REG}_{k}(n) B_{k}
$$

which completes the proof. 
We will call the vectors $\left(B_{n}\right)_{n \in \mathbb{N}} \in \mathbb{Z}^{N}$ appearing in the formula (36) the vector Dold coefficients.

In connection to exact realizable sequences, we define their more general counterpart (remind that we assume that the conditions (A1)-(A3) are satisfied).

Definition 29. A vector valued sequence $\left(a_{n}(i)\right)_{n}, 1 \leqslant i \leqslant N, n \in \mathbb{N}$, such that $a_{n}(i) \in$ $\mathbb{N} \cup\{0\}$ will be called exactly realizable for the partial order $P$ if there is a space $X$ and a map $f: X \rightarrow X$ satisfying: $a_{n}(i)=\# \operatorname{Fix}_{i}\left(g^{n}\right)(\mathrm{cf}$. Remark 23$)$ for all $n \geqslant 1, i=1, \ldots, N$.

Corollary 30. A vector sequence $\left(a_{n}(i)\right)_{n}$ is exactly realizable for the order $P$ if and only if $\left(a_{n}(i)\right)_{n}$ is a generalized Dold sequence and all coordinates of vector Dold coefficients $B_{n}(i)$ (given by (36)) are non-negative for every $n$.

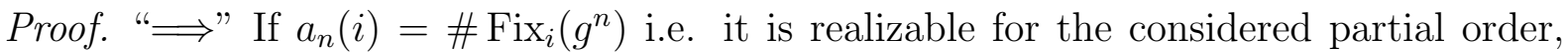
then by Theorem 27 it is a generalized Dold sequence. Furthermore, by (36):

$$
a_{n}(i)=\sum_{k \mid n} \operatorname{REG}_{k}(n) B_{k}(i)
$$

where $B_{k}(i)$ is equal to the number of orbits of length $k$ belonging to the type $i$, thus all $B_{k}(i)$ are non-negative.

"£" For each $i$ and $k$ we take $B_{k}(i)$ orbits of length $k$ and realize $f$ on the discrete set equal to the disjoint sum of orbits.

\subsection{An example of vector Dold sequences}

In this section we discuss a subclass of vector Dold sequences defined for some particular partial orders which appear in Nielsen periodic point theory.

Definition 31. We consider the partial orders $P$ on the set $\mathbb{N} \times\{1, \ldots, N\}$. Let us assume that a map $h_{k n}:\left\{k_{1}, \ldots, k_{N}\right\} \rightarrow\left\{n_{1}, \ldots, n_{N}\right\}$ is given for any $k, n \in \mathbb{N}, k<n$. We assume also that for every $k, n$ such that $k \mid n$ there exist $m$ and $j$ such that $h\left(k_{j}\right)=n_{m}$ and that for every $k, l, n$ such that $k|l| n h_{l n} \circ h_{k l}=h_{k n}$.

We will say that $P$ is $h$-order if it is given by the following relation:

$$
k_{j}<n_{m} \Longleftrightarrow k \mid n \text { and } h_{k n}\left(k_{j}\right)=n_{m}, \quad 1 \leqslant j, m \leqslant N .
$$

Assume that for each natural $n \operatorname{Fix}_{i}\left(f^{n}\right) \cap \operatorname{Fix}_{j}\left(f^{n}\right)=\emptyset$. Then in our model (cf. Remark 23) the condition (41) implies that each fixed point $x_{0}$ has exactly one target type within the set $\left\{n_{1}, \ldots, n_{N}\right\}$ for every $n$.

Let us remark that considering such orders is inspired by the behavior of Nielsen classes of iterations for maps on compact manifolds, where $h_{k n}$ is an inclusion (see [24] for details).

Example 32. Consider the case of $\mathbb{R} P^{n}$. In the set of orbits of Reidemeister classes we define the map induced by inclusion of respective Nielsen classes. If $N^{k} \subset \operatorname{Fix}\left(f^{k}\right)$, 
$N^{n} \subset \operatorname{Fix}\left(f^{n}\right)$ are Nielsen classes representing Reidemeister classes $A^{k} \subset \mathcal{O} \mathcal{R}\left(f^{l}\right)$ and $A^{n} \subset \mathcal{O} \mathcal{R}\left(f^{n}\right)$ respectively, then $N^{k} \subset N^{n}$ implies $h_{k n}\left(A^{k}\right)=A^{n}$.

In the case of self-maps of $\mathbb{R} P^{n}$ of odd degree $\mathcal{O} \mathcal{R}\left(f^{n}\right)$ consists of two elements $\left\{n_{1}, n_{2}\right\}$, and the map $h_{k n}$ defining the order in $P$ is given by the formula:

$$
\begin{gathered}
h_{k n}\left(k_{1}\right)=n_{1} . \\
h_{k n}\left(k_{2}\right)= \begin{cases}n_{2} & \text { if } \frac{n}{k} \text { is odd }, \\
n_{1} & \text { if } \frac{n}{k} \text { is even. }\end{cases}
\end{gathered}
$$

A vector Dold sequence for a given $h$-order $P$ may be characterized in the following way.

Proposition 33. A sequence of vectors $\left(X_{n}\right)_{n \in \mathbb{N}} \in \mathbb{Z}^{N}$ for h-order $P$ is a vector Dold sequence if for each $n \in \mathbb{N}$ and $n_{i}(1 \leqslant i \leqslant N)$

$$
\sum_{k \mid n} \mu(n / k) \sum_{k \in h_{k n}^{-1}\left(n_{i}\right)} X_{k}(i) \equiv 0(\bmod n) .
$$

Proof. First of all let us remark that the only non-zero elements of the matrix $M_{k \rightarrow n}^{*}$ in the formula (34) are equal to $\mu(n / k)$. Indeed, if we define the interval $\operatorname{Int}\left[k_{i}, n_{j}\right]$ as the set of all elements between $k_{i}$ and $n_{j}$, we get that in the $h$-order it is isomorphic (as an order) to the interval $\operatorname{Int}[k, n]$ with the order given by the divisibility relation, i.e. $\operatorname{Int}[k, n]=\{d: k|d| n\}$, which in turn is isomorphic to $\operatorname{Int}[1, n / k]=\left\{d_{1}: d_{1} \mid n / k\right\}$. As a consequence, $\mu^{*}\left(k_{i}, n_{j}\right)=\mu(n / k)$.

On the other hand, for a given row $i$ all non-zero elements of the matrix $M_{k \rightarrow n}^{*}$ are determined by $k \in h_{k n}^{-1}\left(n_{i}\right)$. This completes the proof.

\subsection{Matrix Dold sequences}

Definition 26 of a vector Dold sequence may be naturally modified to matrix Dold sequence.

Definition 34. A sequence of matrices $\left(X_{l}\right)_{l \in \mathbb{N}} \in \operatorname{Mat}_{N \times N}(\mathbb{Z})$ is a matrix Dold sequence if for each $l \in \mathbb{N}$ there exists an integer valued matrix $J_{l} \in \operatorname{Mat}_{N \times N}(\mathbb{Z})$ such that

$$
\sum_{k \mid l} M_{k \rightarrow l}^{*} X_{k}=l J_{l}
$$

The natural corollary from Theorem 28 is the following characterization of matrix Dold sequences.

Theorem 35. The sequence of matrices $\left(X_{n}\right)_{n \in \mathbb{N}} \in \operatorname{Mat}_{N \times N}(\mathbb{Z})$ is a matrix Dold sequence if and only if there exists a sequence of integer matrices $\left(B_{n}\right)_{n \in \mathbb{N}} \in \operatorname{Mat}_{N \times N}(\mathbb{Z})$ such that

$$
X_{n}=\sum_{k \mid n} \operatorname{REG}_{k}(n) B_{k}
$$

for each $n \in \mathbb{N}$. 
Proof. It is enough to observe that the sequence $X_{n}$ is a matrix Dold sequence if and only if for any $j \in\{1, \ldots, N\}$ the sequence of $j$-th columns $X_{n}(\cdot, j)$ is a vector Dold sequence. Moreover, the equation (45) is valid iff the equation (36) is valid for each $j$-th column of $X_{n}(\cdot, j)$ and $B_{k}(\cdot, j)$.

Remark 36. The matrix Dold sequences also may be interpreted in terms of the fixed point theory, similarly as we did in Remark 23, but instead of classifying orbits into finite set of types, we classify them according to two, mutually independent, criteria.

We can think of each orbit as having assigned a type (a number from $\{1, \ldots, N\}$ ) and a color (a number from a finite set $\{1, \ldots, N\}$ ). Then, following the notation similar to the one used in Remark 23, the matrix $A_{k} \in$ Mat $_{N \times N}$ counts the orbits of the length $k$ of the map $g$, where $A_{k}(i, j)$ is a number of orbits having the type $i$ and the color $j$.

Notice also, that fixing the color $j$ we obtain the vector Dold sequence (of $j$-th columns of matrices $A_{k}$ ) corresponding to the set of orbits of fixed color.

\section{References}

[1] I. K. Babenko, S. A. Bogatyi, The behavior of the index of periodic points under iterations of a mapping, Math. USSR Izv. 38:1-26, 1992.

[2] E. Bagno, E. Eisenberg, S. Reches, M. Sigron, On the poset of non-attacking king permutations, European J. Combin. 87:103119, 2020.

[3] R. Brignall, V. Jelinek, J. Kynčl, D. Marchant, Zeros of the Möbius function of Permutations, Mathematika 65(4):1074-1092, 2019.

[4] R. F. Brown, The Lefschetz Fixed Point Theorem, Glenview, New York, 1971.

[5] A. Burstein, V. Jelinek, E. Jelinkova, E. Steingrimsson, The Möbius function of separable and decomposable permutations, J. Combin. Theory Ser. A, 118(8):23462364, 2011.

[6] J. Byszewski, G. Graff and T. Ward, Dold sequences, periodic points, and dynamics, Bull. Lond. Math. Soc. 53(5):1263-1298, 2021.

[7] S. N. Chow, J. Mallet-Parret, J. A. Yorke, A periodic point index which is a bifurcation invariant, Geometric dynamics (Rio de Janeiro, 1981), 109-131, Springer Lecture Notes in Math. 1007, Berlin 1983.

[8] A. Dold, Fixed point indices of iterated maps, Inventiones Math., 74:419-435, 1985.

[9] H.-B. Duan, The Lefschetz numbers of iterated maps, Topology Appl. 67(1):71-79, 1995.

[10] B.-S. Du, S.-S. Huang and M.-C. Li, Generalized Fermat, double Fermat and Newton sequences, J. Number Theory 98:172-183, 2003.

[11] B.-S. Du, S.-S. Huang and M.-C. Li, Newton, Fermat, and Exactly Realizable Sequences, J. Integer Seq. 8(1):05.1.2, 2005.

[12] G. Everest, A. van der Poorten, A. Y. Puri, T. Ward, Integer sequences and periodic points, J. Integer Seq. 5(2):02.2.3, 2002. 
[13] G. Graff, Indices of iterations and periodic points of simplicial maps of smooth type, Topology Appl. 117(1):77-87, 2002.

[14] G. Graff, Minimal number of periodic points for smooth self-maps of two-holed 3dimensional closed ball, Topol. Method Nonl. An. 33(1):121-130, 2009.

[15] G. Graff, J. Jezierski and P. Nowak-Przygodzki, Fixed point indices of iterated smooth maps in arbitrary dimension, J. Differential Equations 251:1526-1548, 2011.

[16] G. Graff, J. Jezierski, Minimal number of periodic points of smooth boundarypreserving self-maps of simply-connected manifolds, Geom. Dedicata 187:241-258, 2017.

[17] G. Graff and P. Nowak-Przygodzki, Fixed point indices of iterations of planar homeomorphisms, Topol. Methods Nonlinear Anal. 22(1):159-166, 2003.

[18] G. Guirao and J. Llibre, Periodic structure of transversal maps on $\mathbb{C} P^{n}, \mathbb{H} P^{n}$ and $\mathbb{S}^{p} \times \mathbb{S}^{q}$, Qual. Theory Dyn. Syst. 12(2):417-425, 2013.

[19] G. Graff, M. Lebiedź, P. Nowak-Przygodzki, Generating sequences of Lefschetz numbers of iterates, Monatsh. Math. 188(3):511-525, 2019.

[20] L. Hernández-Corbato, P. Le Calvez and F. Ruiz del Portal, About the homological discrete Conley index of isolated invariant acyclic continua, Geometry \& Topology 17, 5:2977-3026, 2013.

[21] L. Hernández-Corbato and F. Ruiz del Portal, Fixed point indices of planar continuous maps, Discrete Contin. Dyn. Syst. 35(7):2979-2995, 2015.

[22] P. Le Calvez, F. Ruiz del Portal, J. Salazar, Indices of the iterates of $\mathbb{R}^{3}$ homeomorphisms at fixed points which are isolated invariant sets, J. Lond. Math. Soc. (2) 82(3):683-696, 2010.

[23] J. Llibre and M. Todd, Periods, Lefschetz numbers and entropy for a class of maps on a bouquet of circles, J. Difference Equ. Appl. 11(12):1049-1069, 2005.

[24] J. Jezierski and W. Marzantowicz, Homotopy methods in topological fixed and periodic points theory. Topological Fixed Point Theory and Its Applications, Vol. 3, Springer, Dordrecht, 2006.

[25] P. Le Calvez and J.-C. Yoccoz, Un théoreme d'indice pour les homéomorphismes du plan au voisinage d'un point fixe, Annals of Math. 146:241-293, 1997.

[26] D. G. Mead, Newton's identities, Amer. Math. Monthly 99:749-751, 1992.

[27] G. Minton, Linear recurrence sequences satisfying congruence conditions, Proc. Amer. Math. Soc., 142(7):2337-2352, 2014.

[28] N. Neumärker, Realizability of integer sequences as differences of fixed point count sequences, J. Integer Seq. 12(4):09.4.5, 2009.

[29] OEIS Foundation Inc. (2020), The On-Line Encyclopedia of Integer Sequences, http: //oeis.org

[30] A. Pokrovskii and O. Rasskazov, On the use of the topological degree theory in broken orbits analysis, Proc. Amer. Math. Soc. 132(2):567-577, 2004. 
[31] Y. Puri, T. Ward, Arithmetic and growth of periodic orbits, J. Integer Seq. 4(2):01.2.1, 2001.

[32] A. de Reyna, Dynamical zeta functions and Kummer congruences, Acta Arith. 119(1):39-52, 2005.

[33] G.-C. Rota, On the foundations of combinatorial theory I: Theory of Möbius functions, Zeit. für Wahrscheinlichkeitstheorie 2:340-368, 1964.

[34] B. E. Sagan, V. Vatter, The Möbius function of a composition poset, J. Algebraic Combin., 24(2):117-136, 2006.

[35] M. Scharmach, 2-dimensional Dold sequences, preprint.

[36] V. Sirvent, A Note on the Periodic Structure of Transversal Maps on the Torus and Products of Spheres, Qual. Theory Dyn. Syst. 19(1):\#45, 2020.

[37] J. P. Smith, A formula for the Möbius function of the permutation poset based on a topological decomposition, Adv. in Appl. Math., 91:98-114, 2017.

[38] R. Stanley, Enumerative combinatorics, Vol. I. Wadsworth \& Brooks/Cole Advanced Books \& Software, Monterey, CA, 1986.

[39] E. Steingrimsson, B. E. Tenner. The Möbius function of the permutation pattern poset, J. Comb. 1(1):39-52, 2010.

[40] H. Steinlein, Fermat's little theorem and Gauss congruence: matrix versions and cyclic permutations, Amer. Math. Monthly 124(6) 548-553, 2017.

[41] H. Steinlein, 70 years of asymptotic fixed point theory, J. Fixed Point Theory Appl. 17(1):3-21, 2015.

[42] K. Wójcik, Binomial Transform and Dold Sequences, J. Integer Seq. 18(1):15.1.1, 2015.

[43] K. Wójcik, Newton sequences and Dirichlet convolution, Integers 21:A63, 2021. 